\title{
Amelioration of Stroke-Induced Neurological Deficiency by Lyophilized Powder of Catapol and Puerarin
}

\author{
Yang Liu ${ }^{1,2,3}$, Qiang Xue ${ }^{1,2,3}, \mathrm{Xu} \mathrm{Li}{ }^{1,2,3}$, Jifen Zhang ${ }^{1,2,3}$, Zhifeng Fu4, Binbin Feng 5 , Yi Chen ${ }^{1,2,3}$, Xiaoyu \\ $\mathrm{Xu}^{1,2,3 凶}$ \\ 1. College of Pharmaceutical Sciences \& College of Traditional Chinese Medicine and Pharmacology, Southwest University, Chongqing \\ 400715, China; \\ 2. Institute of Chinese Medicine, Southwest University, Chongqing 400715, China; \\ 3. Chongqing Engineering Research Center for Pharmacological Evaluation, Chongqing 400715, China; \\ 4. Key Laboratory of Luminescence and Real-Time Analysis (Ministry of Education), Southwest University, Chongqing 400715, China; \\ 5. Department of Pharmacology, Chongqing Three Gorges Medical College.
}

$\square$ Corresponding author: Professor Xiaoyu XU, Laboratory of Molecular Pharmacology, College of Pharmaceutical Sciences \& College of Traditional Chinese Medicine, Southwest University, Chongqing 400715, China. Tel.:+0086-23-6825-0761; Fax: +0086-23-6825-1225; E-mail: xuxiaoyu@swu.edu.cn or Yi Chen (Ph.D.), Laboratory of Molecular Pharmacology, College of Pharmaceutical Sciences \& College of Traditional Chinese Medicine, Southwest University, Chongqing 400715, China. E-mail:rachelcy@swu.edu.cn.

(C) Ivyspring International Publisher. This is an open-access article distributed under the terms of the Creative Commons License (http://creativecommons.org/ licenses/by-nc-nd/3.0/). Reproduction is permitted for personal, noncommercial use, provided that the article is in whole, unmodified, and properly cited.

Received: 2014.0I.13; Accepted: 2014.03.22; Published: 20I4.04.08

\begin{abstract}
Catalpol and puerarin are active ingredients isolated from Rehmannia glutinosa Libosch and Radix Puerariae, respectively. They are popular in research for their poly-pharmacological effects. This research focused on effect of anti-stroke by lyophilized powder of catalpol and puerarin (C-P) and potential mechanisms. At the beginning of research, C-P was identified and analyzed by HPLC. Neurological function was evaluated by Longa score, neurological complex function score and beam balance score after permanent middle cerebral artery occlusion (PMCAO) in mice. Infarct volume and water content were evaluated after treatment of C-P. Anti-oxidative stress, anti-apoptosis, angiogenesis and neurogenesis were investigated by ELISA, WB and immunohistochemical stain respectively. With treatment of C-P, neurological deficiency of PMCAO mice was ameliorated. Morphologically, infarct volume and water content in ischemic hemisphere were significantly reduced by C-P. In vivo and in vitro, oxidative stress injury was extenuated by C-P. Meanwhile, Caspase- 3 was down-regulated and $\mathrm{Bxl}-2$ was up-regulated by $\mathrm{C}-\mathrm{P}$ in vivo. In addition, $\mathrm{C}-\mathrm{P}$ enhanced angiogenesis around the infarct of cortex and neurogenesis in the Hippocampal Dentate Gyrus (DG). Hence, C-P ameliorated stroke-induced neurological deficiency through its multiple neuroprotections. What's more, this article provides us a novel formula of active ingredients for stroke.
\end{abstract}

Key words: catapol; puerarin; stroke; neurological function.

\section{Introduction}

Brain stroke imperils human healthy with high risk of fatality and disability [1]. It is mostly resulted from cerebral arterial thrombosis in clinical. After stroke, neural cells suffer from various cellular events which lead cells to apoptosis [2]. Apoptosis is the main pathway that oxidative stress leads cells to death [3]. In the later period of stroke, neural regeneration and plasticity are present as the restorative responses to ameliorate the neurological deficiency [4.]. However, drugs that can target to all these sides are still 
absent. Decades passed with so many studies on stroke, the progress in therapy of stroke is proceeding very slowly. So far, recombinant tissue type plasminogen activator (rt-PA) is the only established clinical therapy for stroke. However, it naturally benefits a small fraction of patients for its limitations, short time window and high risk of rehaemorrhagia [5].

Catalpol is the main active ingredients in Rehmannia glutinosa Libosch which is typically used to treat stroke in china. Its modern research proves that catalpol can improve stroke-induced neurological deficiency of rats [6]. This efficacy is partially related to its angiogenesis, anti-apoptosis and anti-oxidative stress [7-10]. Puerarin is an active ingredient that isolated from Puerariae lobata Ohwi. It has various pharmacological effects as improving microcirculation, anti-platelet aggregation and antithrombotic formation [11]. With reducing infarct volume, it ameliorates stroke-induced neurological deficiency as well [12-16].

Additionally, lots of reports highly suggested that drugs in combination have performed better than alone in neural protection [17]. Although the two active ingredients stated above are effective against brain stroke, there is no any research on them used in combination. Therefore, it provides us a research gap of the two ingredients combined against brain stroke. For clinical use, we have lyophilized catalpol and puerarin for injection. A series of detections in vivo have proved that the lyophilized powder is safety to use, [18-20]. C-P significantly improves the dysfunctions of blood rheology and coagulation in vivo [21], and protects HUVECs from hypoxia/reoxygenation injury [22]. Although lots of studies has been done, the pharmacodynamics researches on brain stroke in vivo are still absent.

This research is aimed to observe the anti-stroke of C-P on mice, including neurological behavior, morphology and potential mechanisms. And the mechanisms will be researched from sides of an-

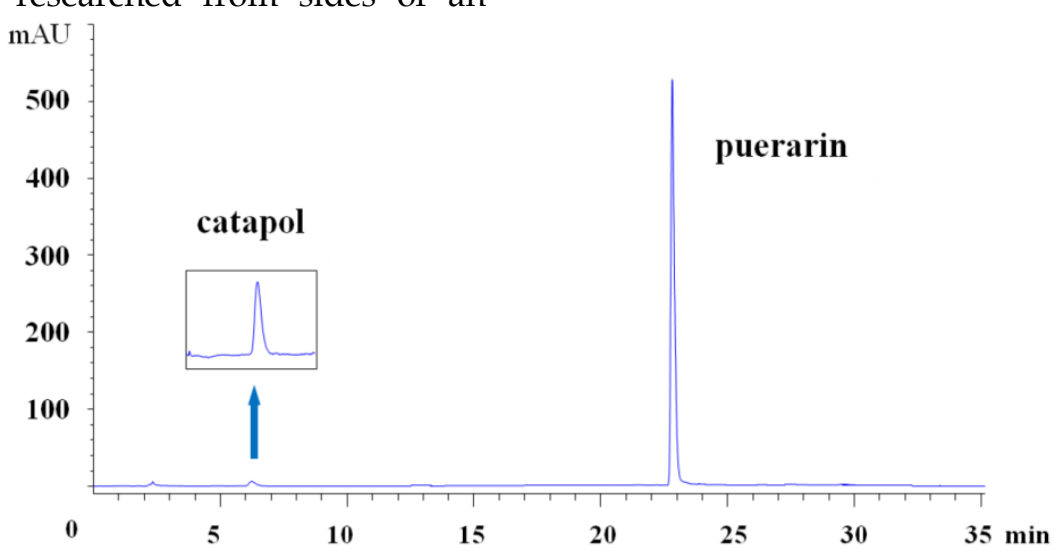

ti-oxidative stress, anti-apoptosis, angiogenesis and neurogenesis by ELISA, WB and immunohistochemical stain.

\section{Materials and Methods}

\section{Reagents}

Catalpol was purchased from Liu bo bai niao Biological Technology Co., Ltd. (China Lot NO. 08051009) and puerarin from $\mathrm{Yu}$ xin Pharmaceutical Co., Ltd. (China Lot NO. 090602). MTT, $\mathrm{CoCl}_{2}$ and $\beta$-Mercaptoethanol were purchased from Sigma (USA). Fetal bovine serum (FBS) was purchased from Hyclone (USA). DMEM was purchased from Gibco (USA). Antibodies of IgG, Caspase-3, $\beta$-actin, CD31, BrdU and Nestin were purchased from Bioss (China). Antibodies of Bcl-2 Bax were purchased from Santa Cruz (USA).

\section{HPLC for C-P}

Chromatographic conditions were as reported before [18]: column Agilent Zorbax SB-C18 (4. 6mm $\times$ $250 \mathrm{~mm}, 5 \mu \mathrm{m}$ ); eluent: water and acetonitrile for gradient elution; the monitoring wavelength was set at $210 \mathrm{~nm}$; flow rate was $1.0 \mathrm{ml} \mathrm{min}-1$ and the column temperature was $30{ }^{\circ} \mathrm{C}$; the sample size was $10 \mu l$. (Fig.1)

\section{Animals and treatment}

Male Kun-Ming mice (25-30g) were purchased from the Experimental Animal Center, Chongqing Medical University (Chongqing, China) and housed under controlled environment $\left(22 \pm 2{ }^{\circ} \mathrm{C}, 12\right.$ hour light/dark cycle, free access to food and water) in the Experimental Animal Center, College of Pharmaceutical Sciences, Southwest University (Chongqing, China). The animal approval number was SYXK 2009-0002. All the experiments were performed in accordance with China's Guidelines for Care and Use of Laboratory Animals.

\footnotetext{
Figure I. High-performance liquid chromatogram of C-P at $210 \mathrm{~nm}$. The first peak is catalpol and the second is puerarin.
} 
Mice were subjected to PMCAO followed Longa's report [23]. 210 stroke mice were randomly divided into PMCOA $(\mathrm{n}=38)$, edaravone (as positive drug, $3 \mathrm{mg} / \mathrm{kg}, \mathrm{n}=38$ ), C-P low (23.4 mg/kg, n=38), C-P middle $(46.7 \mathrm{mg} / \mathrm{kg}, \mathrm{n}=38)$ and C-P high $(93.4$ $\mathrm{mg} / \mathrm{kg}, \mathrm{n}=38$ ) groups. Simultaneously, the extra 38 mice were for sham operation.

Pre-treated with C-P or edaravone (tail-intravenous injection, once a day for 5 days), mice were then subjected to PMCAO. 3 hours later, mice were administered again. And, one hour later, mice were sacrificed for testing the oxidative indexes. In the rest of tests, mice were treated with drugs once a day until being sacrificed for tests. The same volume of medium was for PMCAO and sham group.

\section{PCI 2 cells culture and treatment}

PC12 cells were purchased from Shanghai Life Science Institute of Biochemistry and Cell Biology Research Institute of Chinese Academy of Sciences. Cells were cultured in DMEM/F12 (10\%FBS, Streptomycin $100 \mathrm{mg} / \mathrm{L}$, penicillin $1 \times 10^{5} \mathrm{U} / \mathrm{L}$ ) at the condition of $37^{\circ} \mathrm{C}, 5 \% \mathrm{CO}_{2}$, and the medium were changed every 2-3d.

$\mathrm{CoCl}_{2}$ was used to lead to hypoxia injury [24]. Cells were planted in 96-well plate with concentration of $5 \times 10^{4} \mathrm{ml} / \mathrm{L}$ and divided into groups for control, $\mathrm{CoCl}_{2}$ and C-P (with four gradient: $12.25 \mu \mathrm{g} \mathrm{ml} / \mathrm{L}$, $24.5 \mu \mathrm{g} \mathrm{ml} / \mathrm{L}, 49 \mu \mathrm{g} \mathrm{ml} / \mathrm{L}$ and $98 \mu \mathrm{g} \mathrm{ml} / \mathrm{L}$ ). Treated with C-P for 1 hour, PC12 cells were co-cultured with $\mathrm{CoCl}_{2}$ (500umol/L) and C-P for $24 \mathrm{~h}$.

\section{Behavioral test}

72 Mice $(n=12)$ were tested by Longa score [23], neurological complex function [25], and beam balance test [26]. All animals were pretrained for 7 day before PMCAO, and then tested on the 1th, 4th and 7th day after stroke.

\section{Infarct volume}

48 brains $(n=8)$ were for TTC stain on the 7 th day after stroke. Brains were taken out without olfactory bulb and brainstem, and then put them into the Brain Matrices (1MM 175-300). Every brain was cut for 4 coronal slices ( $2 \mathrm{~mm}$ thick) along the chiasma opticum. The slices were immersed in 2\% TTC for 15 minutes, and fixed in $4 \%$ paraformldehyde overnight [27]. Infarct volume was expressed as a percentage of the whole brain.

\section{Water content}

48 mice $(n=8)$ were sacrificed on the 3 th day. The wet weight (WW) and dry weight (DW) of ischemic hemisphere were recorded [28]. Percentage of water content was calculated as follows: water (\%) = $(\mathrm{WW}-\mathrm{DW}) / \mathrm{WW} \times 100 \%$.

\section{ELISA for oxidative indexes}

Brain specimen

48 mice $(n=6)$ were sacrificed for ELISA at $4 \mathrm{~h}$ and on 1th, 4 th and 7 th after stroke. Brains were made into $10 \%$ brain homogenate and centrifuged $\left(1500 \mathrm{~g} \times 10\right.$ minutes) at $4^{\circ} \mathrm{C}$. The supernatant liquid was detected by ELISA for MDA, NO and SOD.

\section{Cells specimen}

Cells were washed twice with PBS, lyzed on ice for 15 minutes and centrifuged $(1200 \mathrm{~g} \times 10$ minutes $)$ at $4^{\circ} \mathrm{C}$. Supernatant liquid was taken out for detection of SOD. The cell culture medium was used for the test of NO activity. All ELISA steps were followed as the instructions of reagent kit.

\section{BrdU injection}

4 animals were received IP of Bromo-deoxy-Uridine (BrdU: $50 \mathrm{mg} / \mathrm{kg} \mathrm{q}, 12$ hours) on days 1 to 7 post-op to label dividing cells [29].

\section{Immunohistochemistry}

Immunohistochemistry was tested on the 1th day, 4th day and 7th day. All mice were anaesthetized before specimen preparation. Thoracic cavity was opened for exposure of heart. Push catheter to aortic arch and then fixed it. Cold physiological saline was poured into auricula dextra by constant-flow pump until the crystal liquid flowing out from auricula dextra. Then paraform was injected until the all fours were stiff. Brains were taken out without olfactory bulb and epencephala, and fixed in $4 \%$ paraform at room temperature for 3 days. Paraffin sections $(6 \mu \mathrm{m}$ thickness) were for immunohistochemical stains.

Concentrations of various antibodies were used as follows: CD31 polyclonal antibody (1:100), Nestin polyclonal antibody $(1: 100)$ and BrdU monoclonal antibody (1:100). The procedure was followed as previous reports [30]. The positive cells in brain slices were counted with Image-Pro Plus 6.0.

\section{Microvascular density (MVD)}

CD31 was used to mark endothelial cell and evaluate the MVD. The $\mathrm{CD} 31^{+}$cells apart from the vessel, no matter alone or in groups, would be regarded as a microvessel and should be counted. 6 slices of a group were counted and the number of microvessels was counted in five sights of $400 \times$ field for calculating the MVD (number of microvessels per $\mathrm{mm}^{2}$ ) and [31].

\section{MTT}

PC12 cells were incubated with MTT medium $(5 \mathrm{mg} / \mathrm{ml})$ in $96-$ well plate for $4 \mathrm{~h}\left(37^{\circ} \mathrm{C}, 5 \% \mathrm{CO} 2\right)$. The medium was abandoned carefully, and then DMSO to 
solve the formazan (150 $\mu 1$ per well of 96-well plate). Then the absorbance was detected at $490 \mathrm{~nm}$ by Microlab F.A.M.E.

\section{Western Blot (WB)}

Antibodies: IgG/Horseradish peroxidase labeled (1:20000), Bax polyclonal antibody (1:500), Bcl-2 polyclonal antibody (1:500), Caspase-3 polyclonal antibody (1:1700) and $\beta$-actin (1:1000). And the protocol followed as previous report [32].

\section{Statistical analysis}

All of the experiments were repeated at least three times. Quantitative data were expressed as mean \pm SD and analyzed by one-way analysis of variance (ANOVA) with SPSS 17.0. $P<0.05$ was considered statistically significant. All pictures were processed by origin 8.0 and Adobe Illustrator CS5.

\section{Results}

\section{C-P ameliorated stroke-induced neurological deficiency}

Sham group was absent of neurological deficiency. Symptoms of brain stroke were expressed in PMCAO mice, as lassitude, decreased of taking food and weight, hard to breathe and high risk of death during the former $48 \mathrm{~h}$. Presence of pursuing tail and curled foreleg on the opposite of ischemic side could be observed as well. Only those who got scores more than 1 would be served as successful PMCAO model [23]. Longa score, neural complex function and beam balance score were tested for each mice. Mice those treated with C-P or Edaravone expressed less neurological deficiency. The C-P-induced amelioration was mainly initiated from the 4th day, especially with middle dose (Fig.2, $P<0.01$ or $P<0.05$ ).

\section{C-P reduced infarct volume and water content}

On the 7th day of stroke, infarct volume was tested. Compared with PMCAO group, the infarct volume of C-P groups was significantly reduced and almost equal to edaravone group (Fig.3A, $P<0.01$ ). Further, water content in the ischemic hemisphere was obviously decreased by C-P on the 3th day when stroke-induced edema was severe [28] (Fig.3B, $P<0.01)$.

\section{C-P performed anti-oxidative stress and anti-apoptosis}

The anti-oxidative stress and anti-apoptosis of C-P were researched in this section. With treatment of C-P, the oxidative stress injury was weakened in vitro and in vivo. In vivo, the SOD was enhanced significantly by C-P during 4hours-7days, while the NO and MDA were decreased (Fig. $4, P<0.05$ or $P<0.01$ ). In vitro, C-P survived the $\mathrm{CoCl}_{2}$-damaged PC12 cells. Simultaneously, it increased SOD and decreased NO as well (Fig.5, $P<0.05$ or $P<0.01$ ).

Furthermore, caspase- 3 , Bax and Bcl-2 were assayed by WB in $\mathrm{CoCl}_{2}$ damaged PC12 cells. Significantly, rate of Bax/Bcl-2 was decreased by C-P which mainly depended on up-regulation of Bcl-2(Fig.6A, $P<0.05)$. Further, caspase-3 was also decreased obviously (Fig.6B, $P<0.01$ ).
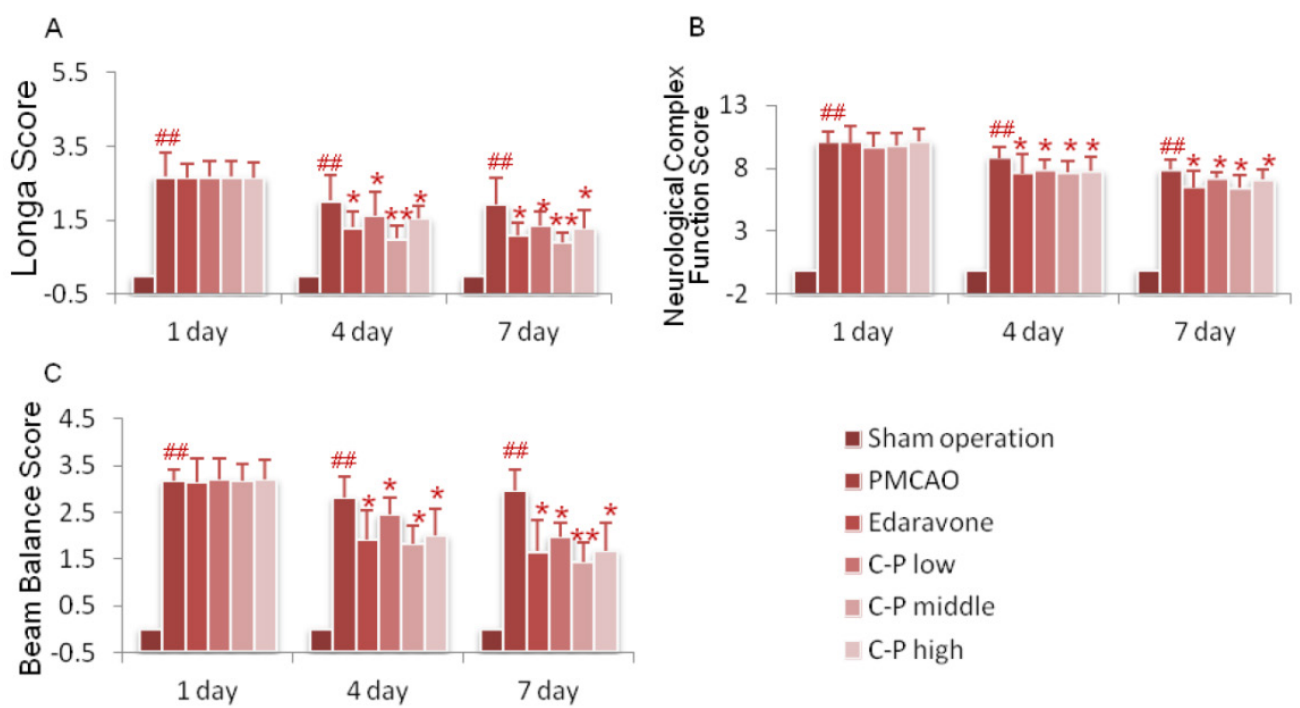

Figure 2. Longa score (A), neurological complex function score (B) and beam balance score $(C)$ were tested in PMCAO mice on the Id, $4 \mathrm{~d}$ and $7 \mathrm{~d}$. (value as mean $\pm S D, n=12,{ }^{\#} P<0.01$ vs sham operation; ${ }^{*} P<0.05,{ }^{* *} P<0.01$ vs $\left.P M C A O\right)$. 
A
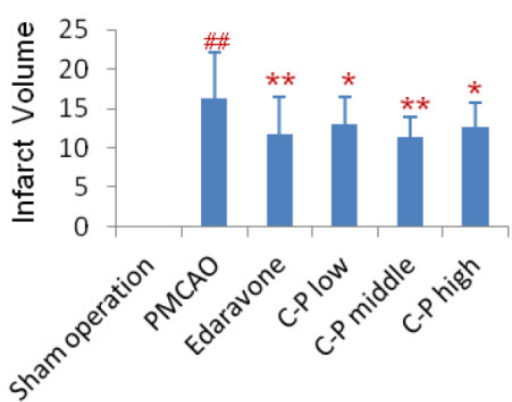

B

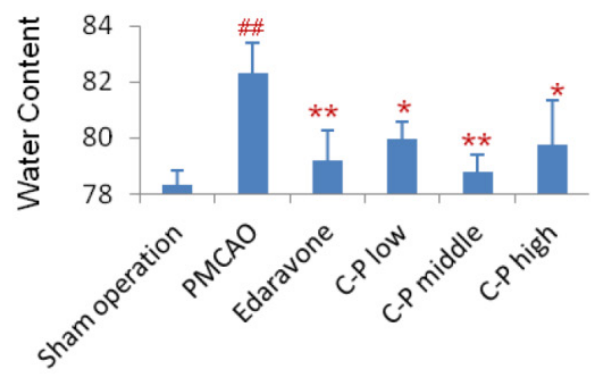

Figure 3. Morphology observation in stroke mice: (A) Treated with C-P for 5 days, mice were performed TTC stain. And the infarct volume was calculated as percentage of infarct volume to whole brain. (B) Water content was tested on the 3 th days of $C-P$ treatment and calculated as follows: water content(\%) $=$ (wet weight - dry weight) $/$ (wet weight). (value as mean $\pm \mathrm{SD}, \mathrm{n}=8,{ }^{\# *} P<0.01$ vs sham operation; ${ }^{*} P<0.05,{ }^{* * *} P<0.01$ vs $\mathrm{PMCAO}$ ).

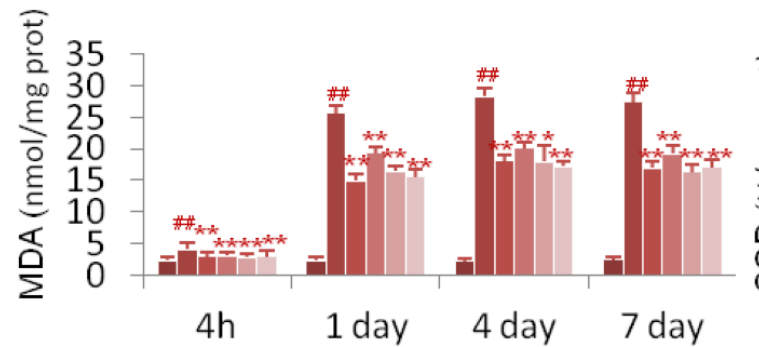

(A)

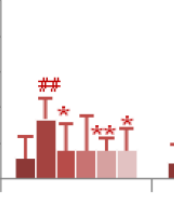

$4 h$

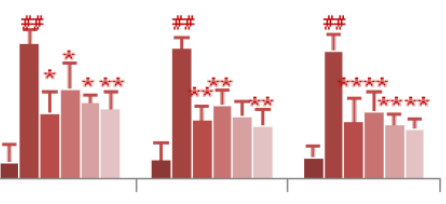

7 day

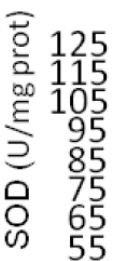

55

$4 h$

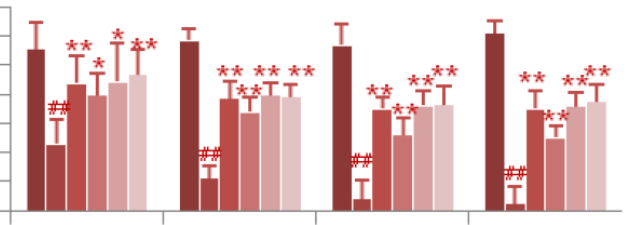

1 day
7 day

(B)

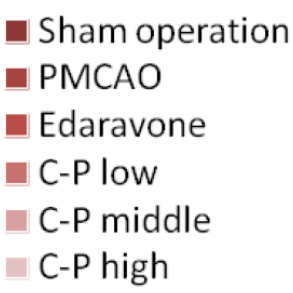

(C)

Figure 4. Anti-oxidative stress of $C-P$ in vivo. PMCAO performed for 3 hours, mice were treated with $C-P$ again. The first test was performed at $4 \mathrm{~h}$ after stroke, and then tested on Ith, 4 th and 7 th respectively. (value as mean $\pm S D, n=6,{ }^{\#} P<0.01$ vs sham operation; ${ }^{*} P<0.05,{ }^{* * *} P<0.01$ vs $P M C A O$ ).

A

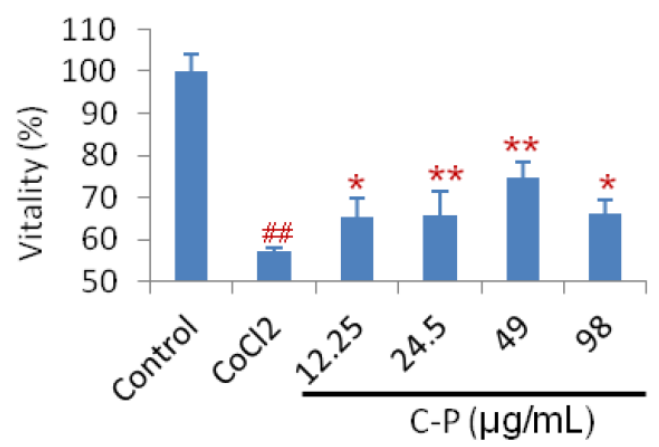

B

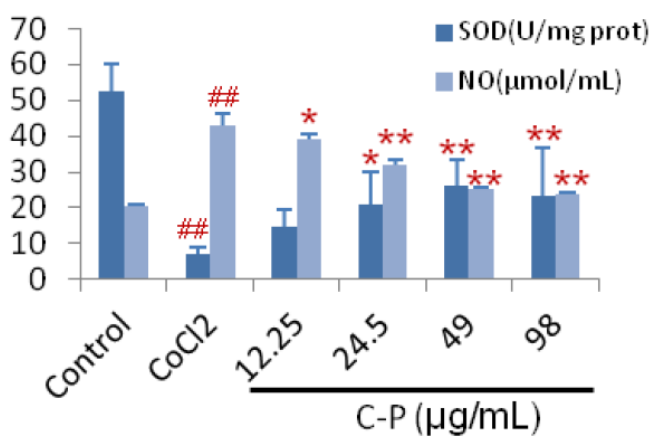

Figure 5. (A) Cells were co-cultured with $\mathrm{CoCl}_{2}$ and C-P for 24 hours, and then the cellular viability was tested by MTT. (B) At the same time, the level of $\mathrm{NO}$ in culture medium and SOD in cells were tested by ELISA. (value as mean $\pm S D, n=3$, repeated to 3 times, ${ }^{\# \prime} P<0.01$ vs sham operation; ${ }^{*} P<0.05$, ${ }^{*} P<0.01$ vs PMCAO). 
A

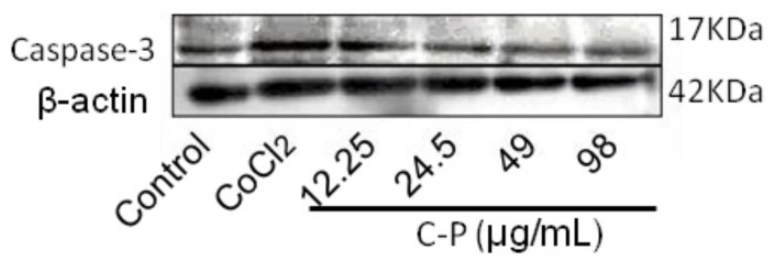

C

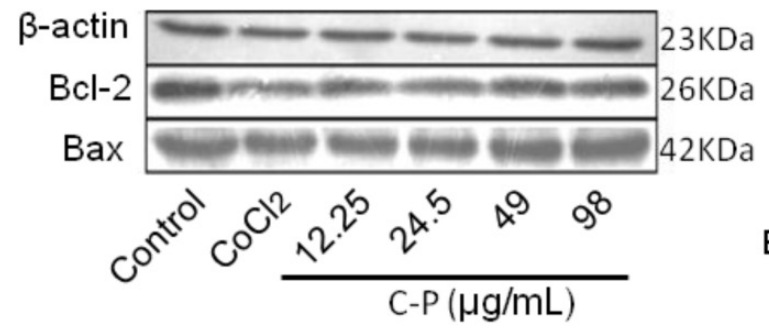

B

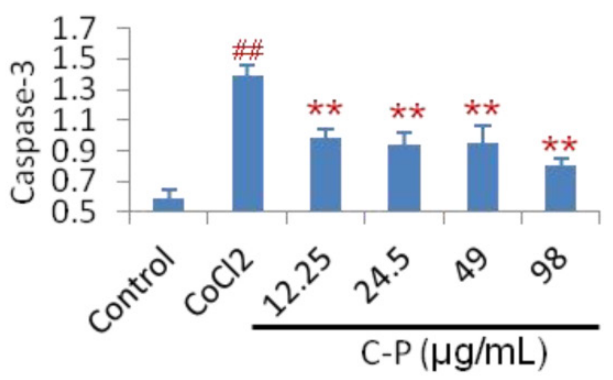

D

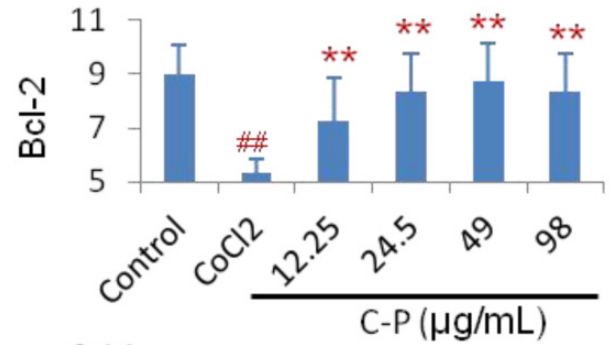

E

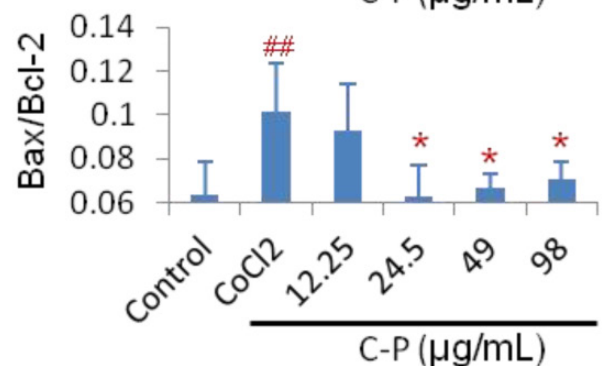

Figure 6. Cells were co-cultured with $\mathrm{CoCl}_{2}$ and $\mathrm{C}-\mathrm{P}$ for 24 hours, and then the expression of $\mathrm{Bcl}-2, \mathrm{Bax}$ and Caspase-3 were tested by WB. Figures show that $\mathrm{C}-\mathrm{P}$ has up-regulated $\mathrm{Bcl}-2$ and decreased caspase-3, but has little effect on Bax. (value as mean $\pm S D, n=3$, repeated to 3 times, ${ }^{\prime} P<0.0 \mathrm{I}$ vs sham operation; ${ }^{*} P<0.05,{ }^{* *} P<0.01$ vs $\left.\mathrm{PMCAO}\right)$.

\section{C-P expanded angiogenesis and neurogenesis}

With increase of BrdU+ cells, the mitotic cells were expanded by C-P in ischemic brains, and mostly initiated from the 1th day after stroke (Fig.7A-E, Fig.8 A-E, $P<0.01$ or $P<0.05)$. This result highly pointed out the enhancement of regeneration in cortex and DG which was benefited from C-P.

Around the infarct of cortex, $\mathrm{CD} 31^{+}$cells in groups of C-P were much more than in PMCAO. Further, the middle dose performed excellently in angiogenesis with the earliest and strongest effect among the three doses (Fig.6D-F, H, $P<0.01$ or $P<0.05)$. In DG of ischemic hemisphere, Nestin ${ }^{+}$cells were expanded by C-P. This neurogenesis was obviously increased from the 4th day (Fig.7 D-F, H, $P<0.01$ or $P<0.05)$.

\section{Discussion}

Brain stroke is a multiple pathologies and targets disease which is hard to be rescued. Deprivation of oxygen and nutrients triggers a complex and interactional cascade of events, as excitotoxicity, calcium overload, oxidative stress, apoptosis, neuroinflammation and so on [2]. And it is the various events that prevent the progress in therapy of stroke. Ameliorating the damage during the ultra-stage of stroke seems promisingly for prognosis. Oxidative stress and apoptosis are predominant pathways that led cells to death in ischemic penumbra which is remediable after stroke [33]. Blocking these two paths is potential to survive the cells in penumbra. On the other hand, regeneration and plasticity are regarded promisingly in stroke, as angiogenesis and neurogensis [4]. All stated above are significant to treat stroke.

Many studies focused on one target of stroke has been failed in vivo or in clinical at last, although them seems effective in vitro [34]. With the good news ceaselessly come from theory of network pharmacology and multiple targets, more and more pharmacologists devote to design new drugs with multiple-efficacies [2, 35, 36]. Under the circumstance, combinative therapy has arisen recently [17]. Combinative therapy not only aims at multiple targets, but also boosts efficacies. Therefore, this paradigm of therapy is prevail in stroke and has obtained some progresses. This combination can be drug therapy with un-drug, as in the case of levodopa with physiotherapy [37]. However, the most of cases are still 
drug with drug in brain stroke. As in the case of rt-PA in combination with aspirin, it is a common and effective treatment against stroke in clinical now. Besides, Candesartan with atorvastatin [38], Memantine with Clenbuterol [39] and Vitamin Dhormone (VDH) with progesterone $(\mathrm{P} 4)$ can protect brain from stroke [40]. Beyond the combination in clinical drugs, drug with peptid can treat acute stroke better as well [41]. In conclusion, the most of combinative drugs are antioxidants, thrombolytics and neuroprotectants.

On the other hand, TCM is hot for its great potential in new drug discovery [42]. Encouragingly, combination of active ingredients from TCM is reported to be potential and promising, as in the two successful cases of therapy in promyelocytic leukemia
[43] and endometriosis [44]. In this study, catalpol in combination with puerarin is because of their inter-enhanced and inter-supplemented efficacies. It is regarded that drugs with qualities of neuroprotection and recovery-enhancement are potential candidates to treat stroke [17]. The previous studies prove that catalpol is prominent in regeneration and plasticity with up-regulation of VEGF, EPO NGF, BDNF and GAP-43 $[7,45]$. It is known to us that the former two factors are prominent in angiogenesis and partially in neurogensis, and the rest is relative to regeneration, growth and plasticity of neurons [7, 8]. Additionally, some other reports indicate that catalpol can regulate energy metabolism [46] and inhibit apoptosis and oxidative stress [9, 10,47, and 48].

Positive cells around the infarction of cortex

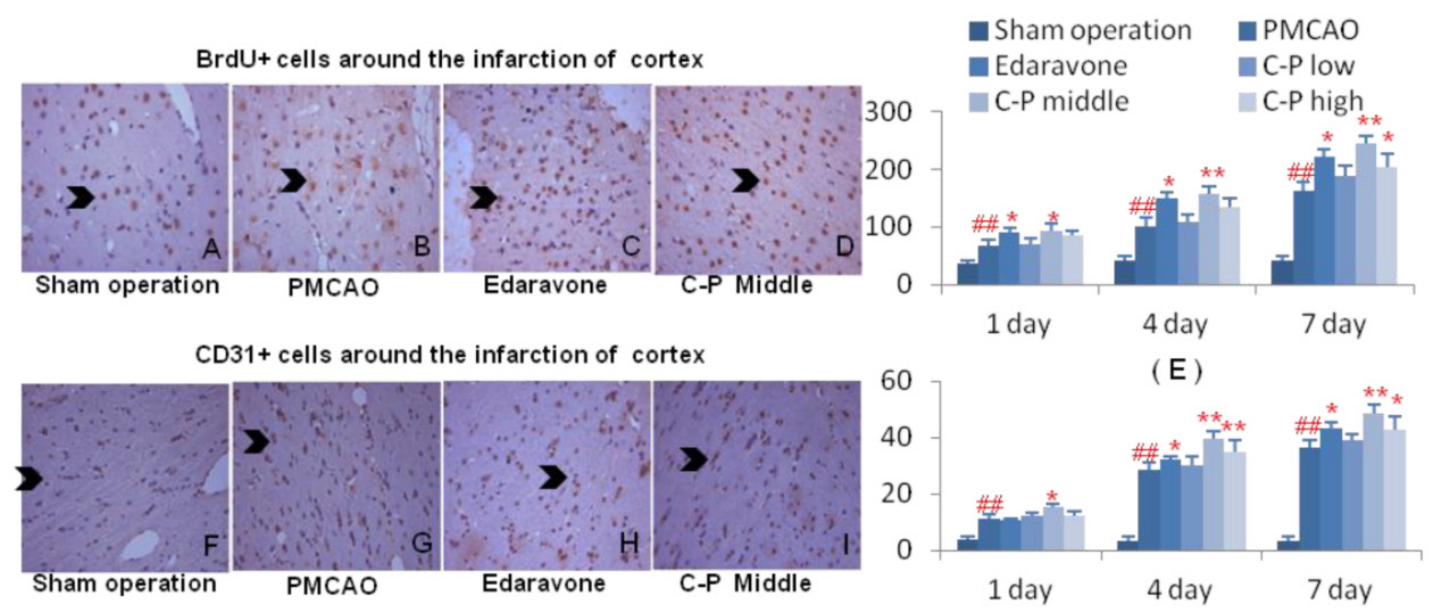

( J )

Figure 7. Aniogenesis around infarct of cortex. Immunohistochemical stains of BrdU and CD3I were performed on the Ith, 4th, 7th day around infarct of cortex. Cells characterized with brown cytoplasm were counted on the specific time $(G-H)$. (value as mean $\pm S D, n=6$, \# $P<0.01$ vs sham operation; ${ }^{*} \mathrm{P}<0.05,{ }^{* *} \mathrm{P}<0.01$ vs $\left.\mathrm{PMCAO}\right)$.
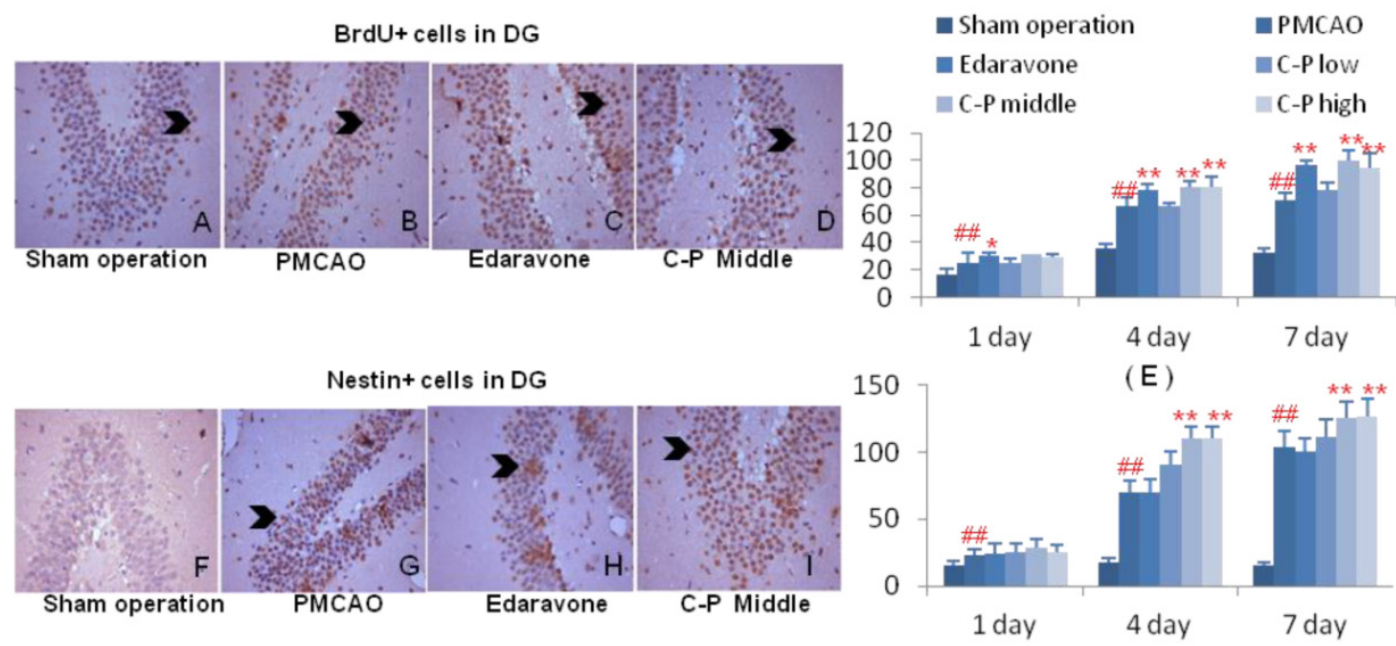

( J )

Figure 8. Neurogenesis in DG of ischemic hemisphere. Immunohistochemical stains of BrdU and Nestin were performed on the Ith, 4th and 7th day after $\mathrm{PMCAO}$ and C-P. Cells characterized with brown cytoplasm were counted $(\mathrm{G}-\mathrm{H})$. (value as mean $\pm \mathrm{SD}, \mathrm{n}=6,{ }^{\#} P<0.01$ vs sham operation; ${ }^{*} P<0.05,{ }^{* * *} P<0.01$ vs PMCAO) 
Recent studies have highly suggested that puerarin is prominent in cardiovascular system: as regulating microcirculation, anti-hypertension, anti-aggregation of platelet and so on [11]. With these efficacies, it is applied into brain stroke and performs excellently not only in acute stroke [49] but also in amelioration of stroke-induced neurological deficiency in clinical [50]. Depending on the sequential studies, the efficacies are likely based on that puerarin protects cells from oxidative stress, DNA damage and apoptosis [13-16]. Also, this effect is observed on endothelial cells, pericytes and neurons [51-53]. Simultaneously, a research indicate that puerarin have induced angiogenesis in myocardium of rat with myocardial infarction [54].

In this article, pretreatment of catalpol with puerarin obviously ameliorated the stroke-induced neurological deficiency. Morphologically, it reduced infract volume and water content of stroke mice. And the mechanisms involved anti-oxidative stress, anti-apoptosis and regeneration. What's more, it provides us two clues: targeting multiple targets by combinative therapy is effective and promising, and TCM is a potential storehouse for drug discovery.

\section{Conclusions}

This article investigated anti-stroke of C-P in pharmacodynamics, as amelioration of stroke-induced neurological deficiency, and induction of infarct volume and water content. These efficacies resulted from anti-oxidative stress, anti-apoptosis and regeneration. However, much more experiments on how to enhance and supply between catalpol and puerarin are still need to be researched intensively in future.

\section{Acknowledgement}

This project was supported by the Ministry of Education Doctoral Program special fund (20110182110012), the Key Projects of Chinese Medicine Research of Chongqing Municipal Health Bureau (2010[60] 2010-1-4), the Program for Innovative Research Team in University of Chongqing (2013), the Projects of Chongqing Science \& Technology (CSTC, 2011jjA0324, 2012jjA1149), and the Southwest University Doctor Foundation (No.SWU111065). At last, we thanks to Jialan Liu and Ling Xu for their special effort on this research.

\section{Competing Interests}

The authors have declared that no competing interest exists.

\section{References}

1. Diener HC, Katsarava Z, Weimar C. Headache associated with ischemiccerebrovascular disease. Rev. Neurol. (Paris). 2008; 164 (10): 819-824.

2. Candelario-Jalil E. Injury and repair mechanisms in ischemic stroke: considerations for the development of novel neurotherapeutics. Current opinion in investigational drugs (London, England: 2000), 2009; 10(7):644-654

3. Chan PH. Role of oxidants in ischemic brain damage. Stroke. 1996; 27(6): 1124-1129.

4. Ohab JJ, Fleming SA, et al. Carmichael. A neurovascular niche for neurogenesis after stroke. The Journal of neuroscience. 2006; 26(50): 13007-13016.

5. Harold P, et al. Guidelines for the early management of patients with ischemic stroke a scientific statement from the stroke council of the american stroke association. Stroke. $2003 ;(34): 1056-1083$.

6. Li DQ, Li Y, Liu Y, et al. Catalpol prevents the loss of CA1 hippocampal neurons and reduces working errors in gerbils after ischemia-reperfusion injury. Toxicon. 2005; 46(8): 845-851.

7. Zhu Hf, Wan D, Luo YP, et al. Catalpol up-regulated GAP-43 protein expression and improved behavior outcome of focal cerebral ischemia rats. Chinese Pharmacological Bulletin. 2007; 23(9): 1231

8. Zhu HF, Wan D, Luo Y, et al. Catalpol increases brain angiogenesis and up-regulates VEGF and EPO in the rat after permanent middle cerebral artery occlusion. International journal of biological sciences. 2010; 6(5): 443-453.

9. Jiang B, Liu J, et al. Catalpol inhibits apoptosis in hydrogen peroxide-induced PC12 cells by preventing cytochrome $<\mathrm{i}>\mathrm{c}$ release and inactivating of caspase cascade. Toxicon. 2004; 43(1): 53-59.

10. Bi J, Jiang B, Hao S, Zhang A, et al. Catalpol attenuates nitric oxide increase via ERK signaling pathways induced by rotenone in mesencephalic neurons. Neurochemistry international. 2009; 54(3): 264-270.

11. Li $Y$, Pan $\mathrm{W}$, Chen $\mathrm{S}, \mathrm{Xu}$ HD, et al. Pharmacokinetic, tissue distribution, and excretion of puerarin and puerarin-phospholipid complex in rats. Drug development and industrial pharmacy. 2006; 32(4): 413-422.

12. Chang $Y$, et al. Neuroprotective mechanisms of puerarin in middle cerebral artery occlusion-induced brain infarct in rats. J Biomed Sci. 2009; 16(9): 1-13.

13. $\mathrm{Xu} \mathrm{X}$, et al. The Neuroprotection of puerarin against cerebral ischemia is associated with the prevention of apoptosis in rats. Planta medica, 2005; 71(7): 585.

14. Wan $\mathrm{H}$, et al. Protective effect of chuanxiongzine-puerarin in a rat model of transient middle cerebral artery occlusion-induced focal cerebral ischemia. Nuclear medicine communications, 2008; 29(12): 1113-1122.

15. Liu CM, et al. Puerarin protects the rat liver against oxidative stress-mediated DNA damage and apoptosis induced by lead. Experimental and Toxicologic Pathology, 2012; 64(6):575-582.

16. Tang $\mathrm{XL}$, et al. Dynamic Oxidative Stress and DNA Damage Induced by Oestrogen Deficiency and Protective Effects of Puerarin and $17 \beta$-Oestradiol in Ovariectomized Rats. Basic \& clinical pharmacology \& toxicology, 2012;111(2):87-91.

17. Fisher M. New approaches to neuroprotective drug development. Stroke, 2011; $42(1$ suppl 1): 24-27.

18. Yuan HH, Zhang JF, Wang Q, Deng LJ, Xu XY. Focal irritant, allergia, haemolyticus and acute toxicity of Catalpol and Puerarin for injection. Chinese Pharmacological Bulletin. 2013; 29(6): 880-881

19. Yuan HH, et al. Determination of Catalpol and Puerarin Contents and Their Related Substances in Zige Freeze-Dried Powder for Injection by RP-HPLC. Chinese Pharmaceutical Journal 2011; 16: 020.

20. Wang $Q$, Xing $M$, Deng $L$, et al. Distribution of puerarin contained in zige freeze-dried powder injection in rat liver and kid-ney. China journal of Chinese materia medica. 2012; 37(11): 1677.

21. Deng $\mathrm{L}$, Wang $\mathrm{Q}$, Yuan $\mathrm{H}$, et al. Effect of catalpol and puerarin freeze-dried powder on coagulability, hemorheology and no in rats with Qi-deficiency and blood-stasis syndrome. China journal of Chinese materia medica. 2012; 37(10): 1472.

22. Zhang $\mathrm{C}, \mathrm{Ma} \mathrm{Q}, \mathrm{Xue} \mathrm{Q}$, et al. Protective effect of Zige lyophilized powder on hypoxia/reoxygenation injury of human umbilical vein endothelial cells. China journal of Chinese materia medica. 2012;37(7):997.

23. Longa EZ, Weinstein PR, et al. Reversible middle cerebral artery occlusion without craniectomy in rats. Stroke. 1989; 20(1): 84-91.

24. Wang GL. and Semenza G. Characterization of hypoxia-inducible factor 1 and regulation of DNA binding activity by hypoxia. Journal of Biological Chemistry.1993;268(29): 21513-21518.

25. Petullo D, Masonic K, Lincoln C, Wibberley L, et al. Model development and behavioral assessment of focal cerebral ischemia in rats. Life sciences. 1999;64(13): 1099-1108.

26. Chen J, Sanberg PR, Li Y, et al. Intravenous administration of human umbilical cord blood reduces behavioral deficits after stroke in rats. Stroke. 2001; 32(11): 2682-2688.

27. Bederson J, Pitts L, Germano S, Nishimura M, Davis R, Bartkowski H. Evaluation of 2, 3, 5-triphenyltetrazolium chloride as a stain for detection and quantification of experimental cerebral infarction in rats. Stroke. 1986; 17: 1304 -1308 .

28. Ito U, Ohno K, Nakamura R, et al. Brain edema during ischemia and after restoration of blood flow. Measurement of water, sodium, potassium content and plasma protein permeability. Stroke. 1979; 10(5): 542-547.

29. Ronen R, et al. Long-Lasting Regeneration after Ischemia in the Cerebral Cortex. Stroke.2007; 38:153-161. 
30. Lixin Sun, Jeongwu Lee and Howard Fine A. Neuronally expressed stem cell factor induces neural stem cell migration to areas of brain injury. J Clin Invest. 2004; 113(9):1364-1374

31. Weidner N, Semple JP, et al. Tumor angiogenesis and metastasis - correlation in invasive breast carcinoma. New England Journal of Medicine. 1991; 324(1): $1-8$.

32. $\mathrm{Q}$ Xue, et al. A Novel Brain Neurovascular Unit Model with Neurons, Astrocytes and Microvascular Endothelial Cells of Rat. Int J Bio Sci. 2013; 9(2):174-189

33. Dirnagl U, et al. Pathobiology of ischaemic stroke: an integrated view. Trends in neurosciences. 1999; 22(9): 391-397.

34. O'Collins V.E, et al. Preclinical drug evaluation for combination therapy in acute stroke using systematic review, meta-analysis, and subsequent experimental testing. Journal of Cerebral Blood Flow \& Metabolism, 2011;31(3):962-975.

35. Barabási, AL, Gulbahce $\mathrm{N}$, and Loscalzo J. Network medicine: a net-work-based approach to human disease. Nature Reviews Genetics, 2011;12(1):56-68

36. Gladstone DJ, et al. Toward wisdom from failure lessons from neuroprotective stroke trials and new therapeutic directions. Stroke, 2002; 33(8):2123-2136.

37. Scheidtmann K, et al. Effect of levodopa in combination with physiotherapy on functional motor recovery after stroke: a prospective, randomised, dou-ble-blind study. The Lancet, 2001; 358(9284): 787-790.

38. Gaur V, and Kumar A. Neuroprotective potentials of candesartan, atorvastatin and their combination against stroke induced motor dysfunction. Inflammopharmacology, 2011; 19(4):205-214.

39. Culmsee $C$, et al. Combination therapy in ischemic stroke: synergistic neuroprotective effects of memantine and clenbuterol. Stroke, 2004;35(5): 1197-1202.

40. Atif $\mathrm{F}$, et al. Combination treatment with progesterone and vitamin D hormone is more effective than monotherapy in ischemic stroke: the role of BDNF/TrkB/Erk1/2 signaling in neuroprotection. Neuropharmacology, 2013; 67:78-87.

41. Zhang L, et al. Combination Treatment With N-Acetyl-Seryl-Aspartyl-Lysyl-Proline and Tissue Plasminogen Activator Provides Potent Neuroprotection in Rats After Stroke. Stroke, 2014; STROKEAHA. 113.004399.

42. Gong $X$, and N.Sucher J. Stroke therapy in traditional Chinese medicine (TCM): prospects for drug discovery and development. Trends in pharmacological sciences, 1999; 20(5):191-196.

43. Wang L, et al. Dissection of mechanisms of Chinese medicinal formula Realgar-Indigo naturalis as an effective treatment for promyelocytic leukemia. Proceedings of the National Academy of Sciences, 2008; 105(12):4826-4831.

44. Tang $\mathrm{Q}$, et al. Combination use of ferulic acid, ligustrazine and tetrahy-dropalmatine inhibits the growth of ectopic endometrial tissue: A multi-target therapy for endometriosis rats. Journal of ethnopharmacology, 2014

45. LIU M, et al. Catalpol up-regulated NGF, BDNF and mRNA gene expression and improved behavior outcome of rats with ischemic stroke. China Journal of Traditional Chinese Medicine and Pharmacy, 2011; 5: 022.

46. Ming L, et al. Effect of catalpol on brain energy metabolism in rats with permanent cerebral ischemia injury [J]. Pharmacology and Clinics of Chinese Materia Medica, 2010; 5: 019

47. $\mathrm{Hu} \mathrm{L}$, Sun $\mathrm{Y}$, and $\mathrm{Hu}$ J. Catalpol inhibits apoptosis in hydrogen perox-ide-induced endothelium by activating the PI3K/Akt signaling pathway and modulating expression of Bcl-2 and Bax. European journal of pharmacology, 2010; 628(1):155-163.

48. Chen W, et al. Neuroprotective activities of catalpol against CaMKII-dependent apoptosis induced by LPS in PC12 cells. British journal of pharmacology, 2013; 169(5): 1140-1152.

49. Tan Y, Liu M, and Wu B. Puerarin for acute ischemic stroke. Stroke, 2008 . 39(7): 2188-2188.

50. Hu H, Fen F, and Ding M. Effects of puerarin with aspirin on the markers of damaged vascular endothelial cells in patients with acute cerebral infarction]. China journal of Chinese materia medica, 2008; 33(23):2827-2829.

51. Jiang B, et al. Hydrogen peroxide-induced apoptosis in pc12 cells and the protective effect of puerarin. Cell Biology International, 2003; 27(12):1025-1031.

52. Kim J, et al. Puerarin inhibits the retinal pericyte apoptosis induced by advanced glycation end products in vitro and in vivo by inhibiting NADPH oxidase-related oxidative stress. Free Radical Biology and Medicine, 2012; 53(2):357-365.

53. Shi R, and Zhang J. Protective effect of puerarin on vascular endothelial cell apoptosis induced by chemical hypoxia in vitro]. Acta pharmaceutica Sinica, 2003; 38(2):103.

54. Zhang S, et al. Puerarin induces angiogenesis in myocardium of rat with myocardial infarction. Biological and Pharmaceutical Bulletin, 2006; 29(5):945. 